\title{
A Web-based Visual Simulator with Communication Support and its Application to Computer Architecture Education
}

\author{
Yoshiro Imai*, Keiichi Kaneko** and Masaki Nakagawa** \\ * Kagawa University ** Tokyo University of Agriculture and Technology \\ E-mail:*imai@eng.kagawa-u.ac.jp,**\{k1kaneko,nakagawa\}@cc.tuat.ac.jp
}

\begin{abstract}
This paper presents development of a web-based visual simulator with communication support and its application to computer architecture education. The simulator written in Java has equipped visualization and communication support for real education. The former is designed to show how a computer works graphically and helps learners to understand basic computer architecture visually. The latter is realized with a built-in e-mail function. It allows learners to send question e-mails with a snapshot of simulator's execution to instructors. They receive such question emails and then redo to verify the execution of learners' simulators. With these supporting equipments, learners and their instructors can share suitable information between their simulators.
\end{abstract}

\section{Introduction}

Recently, an IT-based education becomes one of the most essential and valuable themes for almost all instructors and learners in many kinds of fields, especially engineering field. So it must be necessary for learners to understand computer literacy in the efficient way [2][3][4]. Of course, it takes relatively long time for beginners to study how a computer works. Several kinds of simulators have been designed and developed to educate computer literacy, information science and computer system as educational tools [1]. Using a web-based educational tool is one of the most efficient and effective approaches [3]. Many researches and projects have provided useful tools during a few years. So-called eLearning seems to be one of the most fruitful results of education with these web-based tools.

Visualization is an absolutely necessary keyword and idea to improve the learner's understanding level [3][4]. For example, when teachers educate their students about computer, they want to use efficient educational tools. These tools are expected to have some kind of facility concerning visualization. With such tools, many students will understand computer in a shorter period than the case without using visualization tool [2][3][4].

The second but not least significant keyword is communication support [3]. Learners sometimes want to communicate with instructors and instructors want transmit some kind of information to their learners. It is also important how to realize a facility of communication support in an educational tool.

\section{Design of a Web-based Educational Tool}

In order to learn computer system, especially computer architecture, it is very important to understand the internal behavior and structure of computer. In such a case, it is effective for a learner to utilize an educational tool with visualization facility. For example, a learner uses the educational tool to simulate a sample program graphically in the registertransfer level. If a learner wants to know a role of a specific register, PC (Program Counter), he or she can stop the simulation at any point, change the value of $\mathrm{PC}$, and then restart the simulation in the registertransfer level. An effect of simulation increases more and more with such a visualization facility[2][3][4].

On-line help function is necessary for learners to use the educational tool by themselves. Instructors can prepare the some information about tool for learners through message of online help. For the sake of providing a suitable online-help message, the educational tool itself can get such a message from instructors' side; for example, a Web-based tool gets a text of message from its Web server by means of HTTP connection. In the same manner, the Web-based educational tool can get sample programs from its Web server according to request of its user. Such a mechanism is very good for instructors to provide sample programs suitable for the understanding level of each learner and deliver them to the learner at any time.

It is useful that such an educational tool will equip facility of communication support at the same time. For example, an instructor receives request message or 
question from a learner by means of such communication support, illustrates (i.e. educates visually) how to resolve the problem for the relevant learner and then distributes the according result into other learners as a real example also by means of communication. Such a facility of communication support is very effective and applicable to utilize communication between learner and instructor as well as between learners. It will be one of the potential powers to promote collaborative learning.

A Web-based educational tool is strongly expected to easily distribute to the users and work independently from the limitation of executing environment. An educational tool for real computer education must be designed not only to understand the behavior and structure of computer but also to provide a facility for assembly programming. In addition, it is necessary to equip a smart communication facility in the programming environment in order to realize smooth information exchanging between pair of students without disturbance for others. Such an environment can provide GUI function with visualization to users.

\section{A Web-based Visual Simulator with Communication Support}

In this section, the overview of our visual computer simulator is illustrated as a Web-based educational tool, which is designed and developed not only with a function of Visualization but also with a function of communication support.

\subsection{Visualization Support}

Our visual computer simulator called VisuSim is designed for an educational tool to demonstrate how a computer works graphically. Figure 1 shows an overview of VisuSim and its GUI as a Java applet downloaded from a dedicated Web server. The server includes a Java-applet code for VisuSim, several kinds of sample program and on-line messages for its users.

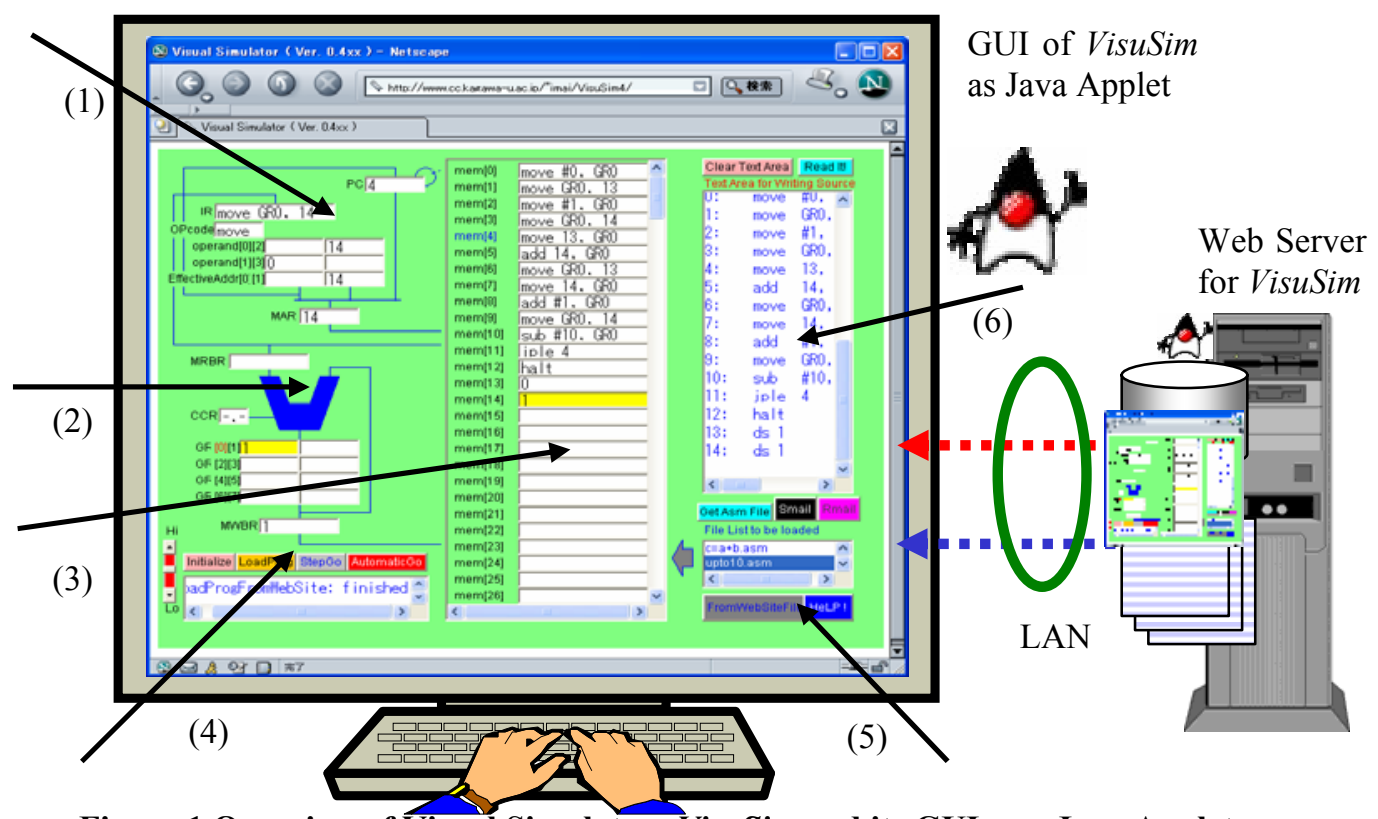

Figure 1 Overview of Visual Simulator: VisuSim and its GUI as a Java Applet

The GUI of VisuSim is organized with following parts and buttons. The GUI facilitates to illustrate how a computer works and gives user (i.e. learner) of VisuSim graphical explanation of internal behavior and structure of computer.

(1) of Figure 1 shows Control Unit part of VisuSim: This part includes Program counter:PC, Instruction register:IR, Opcode decoder and special fields for effective addressing. It illustrates the instruction fetching, its decoding, generating effective address and updating PC. It also explains how the instruction of memory whose address is specified by $\mathrm{PC}$ is transferred from memory into IR and then it is decoded to generate effective address.

(2) of Figure 1 shows Processing Unit part of VisuSim: This part includes Arithmetic Logical Unit, Condition code register:CCR and General registers: GRs. It calculates unary operation and binary operation and generates according result and the program state into GRs and CCR respectively. The data of memory 
whose address is specified by Memory address register is transferred from memory into Memory data register for reading. And it is calculate with the content of specified GR. When the content of GR is updated, its location has been illustrated by means of changing its color. And the index of GR has been also changed into red character. So users of VisuSim can easily understand which GR has been updated and whether CCR has been changed or not during instruction processing.

(3) of Figure 1 shows Memory Unit part of VisuSim: In order to understand assembly programming more easily and clearly, the contents of memory can be described with assembly codes and/or numerical values. Indications are equipped for learner's convenience as follows: The address of memory which is read has been changed into blue-colored characters. And the location of memory whose content is updated has been detected by means of changing its color of content's frame into yellow.

(4) of Figure 1 shows Control Buttons of VisuSim: These provide functions for initializing VisuSim, loading program from the Web server / a text area for writing program source described later, executing program in the step-by-step mode, and running program in the continuous mode respectively. In order to understand a detail of processing program, users can adjust the execution speed of VisuSim by means of speed control slide bar at the left side of these buttons.

(5) of Figure 1 shows other kinds of control buttons for switching input direction to transfer program and invoking on-line help message. (6) of Figure 1 shows the text area for writing program source. Users write directly their programs in this area. And they can click the control button for switching input direction and copy the content of this area into memory. Finally users can choose to execute their program in the stepby-step mode or run them in the continuous one.

\subsection{Communication Support}

In order to realize communication support between learners and instructors, VisuSim has two following built-in modules for e-mail handling:

- One is an e-mail sending module which works as an SMTP-client.

- Another is an e-mail receiving module working as a POP3-client.

When learners use VisuSim and want to correspond with others, they can invoke e-mail sending or receiving module of VisuSim. These client modules are available whenever VisuSim is invoked as a Java standalone application as well as a Java applet. An SMTPclient is a Simple Mail Transfer Protocol-based sending module, which transfers message from VisuSim to the server.

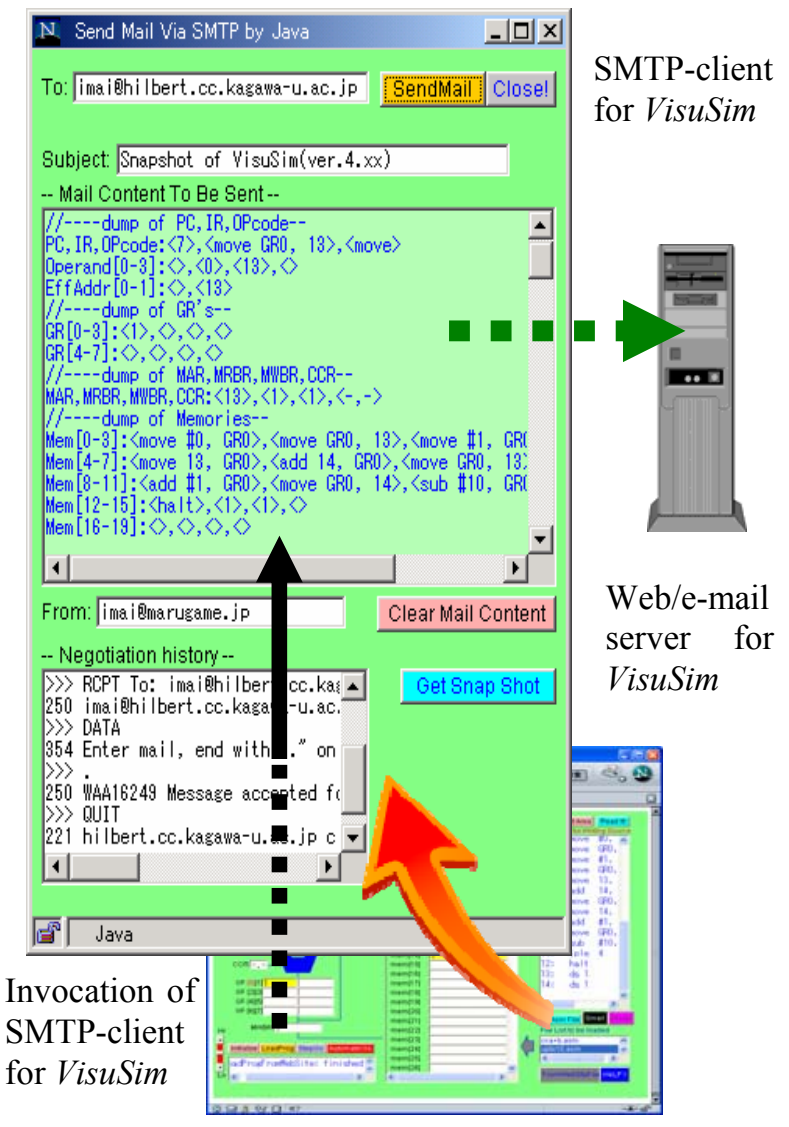

Figure 2 Invocation of STMP-client of VisuSim

VisuSim provides simple information-transmission service which can send a message including the current state of VisuSim, namely, all the contents of registers and memory of itself, to a mail server. With the above service using SMTP-client, VisuSim can make the copy of itself onto another instance of it. There is a particular condition or limitation about capability of SMTP-client. A message from VisuSim must be transferred to the mail server. Namely, VisuSim have to access the external server which is out side of the machine where VisuSim executes. When it works as a Java applet, its SMTP-client should be invoked as a child process of such an applet so that it can only communicate with the mail server which is the same machine of Web server of VisuSim itself. This is a special limitation based on the secure characteristics of Java Applet.

In the case that VisuSim works as a Java stand-alone application, its SMTP-client can communicate with any mail server without such limitation. Figure 2 shows a schematic relation between SMTP-client and VisuSim. This figure illustrates a typical story that a 
learner downloads VisuSim, invokes SMTP-client, adds the current state of VisuSim into an e-mail, and easily send such an e-mail to the mail/Web server.

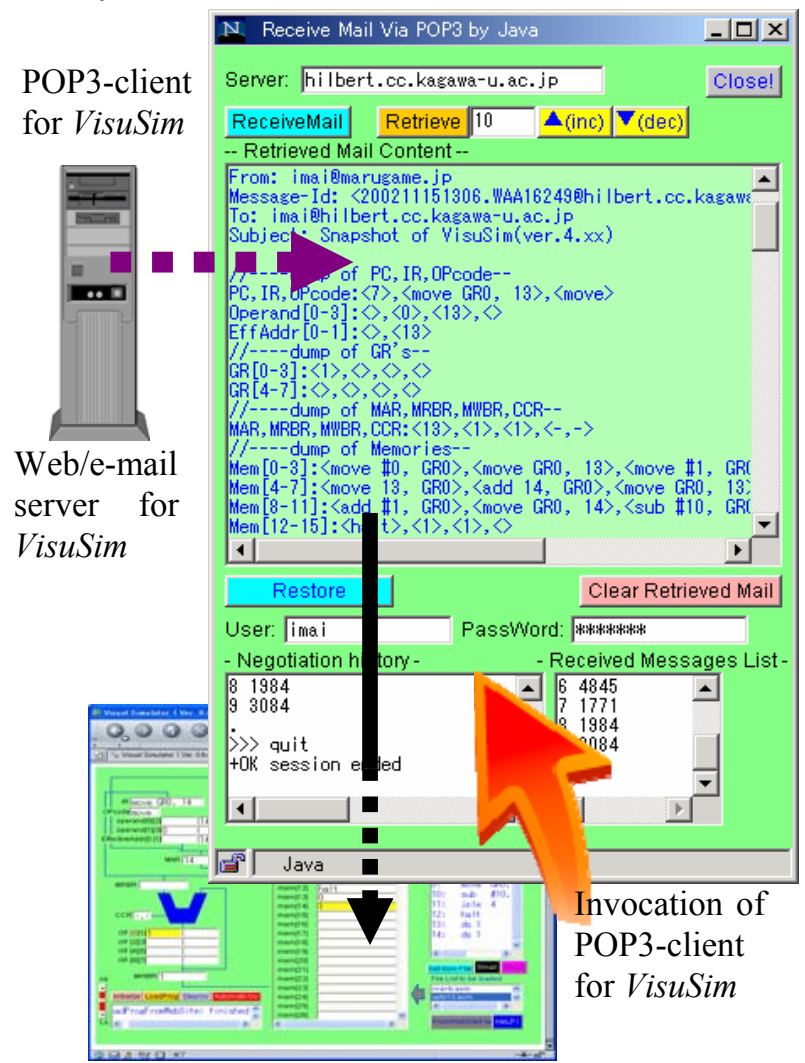

Figure 3 Invocation of POP3-client of VisuSim

On the other hand, a POP3-client is a Post Office Protocol-based receiving module, which obtains information message from the server to VisuSim. Of course, as described above, there is the same condition for POP3-client to access the server just like STMPclient does. Two users of VisuSim can communicate with each other, throw a message from one to another through the intermediary of the mail server, and finally share the same information related to VisuSim between each other. Figure 3 also shows a schematic relation between POP3-client and VisuSim. This figure also illustrates another typical story that an instructor asynchronously downloads VisuSim, invokes POP3client, receives an e-mail with other internal status of VisuSim, restores such a status onto own VisuSim, and shares the same status of other VisuSim of learners.Such facilities are useful to send and receive several kinds of message so that VisuSim can facilitate to share the internal state of VisuSim, namely contents of registers in the specific VisuSim, between some learners of group education in the collaborative learning environment. With these SMTP/POP3-clients, VisuSim can realize communication support, play an effective role of Web-based educational tool for learner

and instructor in the distance and provide a suitable environment for collaborative learning.

\section{Real Computer Education with VisuSim}

VisuSim has been applied into real education from Computer Architecture to Assembly programming. The former is lecture-style education with VisuSim. The latter is self-teaching style one.

\subsection{Computer Architecture Course}

Students of our class can understand the behavior and structure of computer not only through watching demonstration with VisuSim from a teacher but also through manipulation of VisuSim by themselves. It is one of the most popular cases that a teacher of the class of Computer Architecture uses VisuSim as an educational tool in order to explain graphically how a computer works. With a projector and a large screen, the teacher directly gives his students the output of his PC where VisuSim is executing.

At the same time, some students may download VisuSim from a dedicated Web server, execute VisuSim on their PCs and compute (i.e. simulate) some sample programs. In such a case, VisuSim is distributed to students as a form of Java applet. Some sample assembly programs and online-help messages are prepared and downloadable from the same Web server.

For example, one of students may investigate whether his/her program works correctly or not by means of stepwise and/or continuous executing modes of VisuSim. When the student meets the problem and hardly resolves it, he/she requests the teacher to teach how to resolve such a problem. The teacher asks the student to send message with the current state of VisuSim of the student to the mail/Web server.

After the teacher receives a message of e-mail from the student, he gains a resurgence of duplicated state of VisuSim of the student from such a message. So the teacher can realize duplicated image of the remote VisuSim on his local VisuSim and find the reason why he/she cannot resolve such a problem. With use of communication support by VisuSim, results of the problem and resolving process will be shared between the teacher and his students in the class.

Assembly programming exercise is suitable for learners to understand the behavior and structure of computer through their programming process. For example, a pair of learners is obliged to work together to accomplish a common goal under below conditions. 
Learners have to rely on each other to achieve the goal. If any one learner fails to do his or her job part, both of them take the consequences.

Through the process of teaching each other, both skill and knowledge of learners are getting wider and more applicable. And such a collaborative learning will lead to useful teamwork spirits and fruitful cooperative thinking. It is very important for engineers.

\subsection{Brief Evaluation of Real Assembly Programming with Visual Simulator}

A brief evaluation of VisuSim has been done as follows. Learners' achievements are evaluated through assembly programming exercises with VisuSim. Such exercises includes the following 5 themes: 1) simple assembly programs with conditional jump and/or iteration, 2) more complicated ones with double loops, 3) programs with subroutine calling. 4) basic recursive programs, and 5) advanced recursive ones.

The relevant learners are 22 members of the senior class of our engineering faculty. After listening two times of lecture, all of them should try to solve the above problems from 1) to 5) and confirm whether their programs work correctly or not with VisuSim during given two weeks. Of course, it seems that every learner tries to do one's bests for every problem. But easier problems are not so difficult and well achieved, while the later ones are difficult and not so achieved.

For example, the problem related to theme 1) is achieved at the level of almost $79 \%$, although the problem related to theme 5) is achieved at the level of almost 5\%. About more than 50\% learners report that they can utilize VisuSim effectively as their selfappraisal. It has a high tendency that learners who have good score of achievement have reported effective utilization of VisuSim. In addition, a few comments from the learners are introduced as follows:

- With VisuSim, behaviors of computer have been visualized and easy to understand $(73 \%)$.

- With VisuSim, manipulation of array has been illustrated and concretely explained (54.5\%).

By the way, learners who do not achieve at good level have two opinions that assembly programming is very difficult and they cannot clearly understand to utilize VisuSim practically. It is another future problem to be resolved that how to educate improvement of assembly programming exercise and effective utilization of VisuSim.

\section{Conclusion}

Functions and applications of our visual simulator VisuSim are introduced in this paper. VisuSim is designed as a Web-based educational tool to illustrate visually how a computer works. With its facility of GUI and communication support, VisuSim can provide an environment for distance learning for assembly programming. And it may be possibly applied to an actual example of collaborative learning. We can conclude our study as follows:

- With VisuSim, a graphical demonstration is available in classroom lecture on Computer Architecture and Assembly Programming, so that it is easy and transparent for even beginners to understand the internal behavior and structure of computer more precisely.

- Embedded e-mail handler can be provided for communication support between the learners. It is useful for a learner and an instructor to share the status of their VisuSim and exchange question and suggestion between them.

\section{References}

[1] Chung, G.K. et.al.. ,2001. The impact of a simulation-based learning design project on student learning, IEEE Transactions on Education, Vol.44, No.4, pp. 390-398.

[2]Yehezkel,C.,et.al. 2001. Three Simulator Tools for Teaching Computer Architecture, ACM Journal of Educational Resources in Computing, Vol.1, No.4, pp. 60-80 (Dec. 2001).

[3] Llamas,M., et.al. 2001. Simulators over the Network, IEEE Transactions on Education, Vol.44, No.2, p.212, CD-ROM folder 09 (May 2001).

[4] Null, L. and Lobur, J. 2003. MarieSim: The MARIE Computer Simulator, ACM Journal of Educational Resources in Computing, Vol.3, No.2, Article \#1, 29 pages (June 2003). 